\title{
锂离子电池负极材料钛酸锂的研究进展
}

\author{
谭 毅 ${ }^{1,2}$, 薛 冰 ${ }^{1,2}$
}

(大连理工大学 1. 材料科学与工程学院; 2. 辽宁省太阳能光伏系统重点实验室, 大连 116024)

摘 要: 锂离子电池作为一种动力能源, 在电动汽车和各种储能系统中有着良好的应用前景。尖晶石结构的钋酸锂 $\left(\mathrm{Li}_{4} \mathrm{Ti}_{5} \mathrm{O}_{12}\right)$ 负极材料具有较高的脱嵌锂电位平台、优异的循环稳定性、以及突出的安全性能, 被认为是一种非常有 潜力的锂离子电池负极材料, 在锂离子动力电池中具有巨大的发展潜力。然而, 尖晶石型 $\mathrm{Li}_{4} \mathrm{Ti}_{5} \mathrm{O}_{12}$ 存在着本征导 电率低，理论容量小等缺陷，极大地限制了其规模化应用，需要进一步改善和提高。本文总结了尖晶石型 $\mathrm{Li}_{4} \mathrm{Ti}_{5} \mathrm{O}_{12}$ 材料在结构形貌、制备方法和性能方面的研究进展，深入分析和讨论了离子掺杂、碳表面改性和纳米化等改性方法 对尖晶石型 $\mathrm{Li}_{4} \mathrm{Ti}_{5} \mathrm{O}_{12}$ 综合电化学性能的改善效果, 并展望了尖晶石型 $\mathrm{Li}_{4} \mathrm{Ti}_{5} \mathrm{O}_{12}$ 作为锂离子电池负极材料未来的发 展方向。

关 键 词: 钛酸锂; 负极材料; 锂离子电池

中图分类号: TM912 文献标识码: A

\section{Research Progress on Lithium Titanate as Anode Material in Lithium-ion Battery}

\author{
TAN Yi ${ }^{1,2}$, XUE Bing ${ }^{1,2}$
}

\begin{abstract}
(1. School of Materials Science and Engineering, Dalian University of Technology, Dalian 116024, China; 2. Key Laboratoryfor Solar Energy Photovoltaic System of Liaoning Province, Dalian 116024, China)
\end{abstract}

\begin{abstract}
With the increasing demand for light, small, high power rechargeable lithium ion batteries in the application of mobile phones, laptop computers, electric vehicles, hybrid electric vehicles, electrochemical energy storage, and smart grids, the development of anode materials with high safety, environmental benignity, high power density, and long cycle life is in progress in recent years. The spinel lithium titanium oxide $\left(\mathrm{Li}_{4} \mathrm{Ti}_{5} \mathrm{O}_{12}\right)$ anode has become more attractive as alternative anode because of its high safety, abundant titanium dioxide raw materials, stable charge/discharge voltage plateau of $1.5 \mathrm{~V}\left(v s . \mathrm{Li}^{2} / \mathrm{Li}^{+}\right)$, and excellent cycling performance due to its zero-strain volume during charge/discharge process. However, the commercial use of $\mathrm{Li}_{4} \mathrm{Ti}_{5} \mathrm{O}_{12}$ anode material has been hindered by the low intrinsic electronic conductivity. This review focuses on recent studies of the electronic structure and performance, synthesis methods and strategies for improvement in the lithium storage capacity, charge/discharge characteristics, then on its near future development.
\end{abstract}

Key words: $\mathrm{Li}_{4} \mathrm{Ti}_{5} \mathrm{O}_{12}$; anode materials; lithium-ion batteries

锂离子电池作为一种新型化学能源, 由于其具 有循环寿命长、能量密度高、无记忆效应、环境友 好等特点, 迅速发展成为最重要和最先进的二次电 池 ${ }^{[1-2]}$ 。锂离子电池已经被广泛开发应用在一些快速
充电电子产品和电动汽车等领域 ${ }^{[3-4]}$ 。目前, 碳材料 是锂离子电池最常用的商业负极材料，但由于金属 锂与碳电极的电位接近, 快速充电或低温充电易发 生“析锂”现象而引发安全隐患。另外碳负极在脱嵌 
锂离子的过程会发生体积形变, 导致不断有新的活 性点产生, 从而使电池容量下降, 降低寿命。因此, 锂离子电池的发展迫切需要寻求安全可靠、寿命更 长的新型负极来替代碳负极。其中钛酸锂就是这样 一种很有潜力的负极材料。钛酸锂主要包括 $\mathrm{Li}_{4} \mathrm{TiO}_{4}$ 、 $\mathrm{Li}_{2} \mathrm{TiO}_{3} 、 \mathrm{Li}_{4} \mathrm{Ti}_{5} \mathrm{O}_{12}$ 和 $\mathrm{Li}_{2} \mathrm{Ti}_{3} \mathrm{O}_{7}$ 。而 $\mathrm{Li}_{4} \mathrm{TiO}_{4} 、 \mathrm{Li}_{2} \mathrm{TiO}_{3}$ 和 $\mathrm{Li}_{2} \mathrm{Ti}_{3} \mathrm{O}_{7}$ 这三种锂钛氧化物受 $\mathrm{Ti}$ 化合价的影响很 难合成纯相, 给制备工艺带来很大难度, 进而限制 了这三种材料的应用; 尖晶石结构的 $\mathrm{Li}_{4} \mathrm{Ti}_{5} \mathrm{O}_{12}$ (LTO) 凭借安全性高、优良的循环稳定性、平稳的充放电 电压平台等优势, 在电化学领域倍受关注。

\section{$1 \mathrm{Li}_{4} \mathrm{Ti}_{5} \mathrm{O}_{12}$ 的结构与性能}

$\mathrm{Li}_{4} \mathrm{Ti}_{5} \mathrm{O}_{12}$ 是具有 $\mathrm{Fd} \overline{3} \mathrm{~m}$ 空间群和立方对称的尖 晶石结构晶体 ${ }^{[5]}$ 。 $\mathrm{Li}_{4} \mathrm{Ti}_{5} \mathrm{O}_{12}$ 的嵌锂电位在 $1.55 \mathrm{~V}$, 每 个分子单元的 $\mathrm{Li}_{4} \mathrm{Ti}_{5} \mathrm{O}_{12}$ 可以嵌入 3 个 $\mathrm{Li}^{+}$, 对应的理 论比容量为 $175 \mathrm{mAh} / \mathrm{g}$ 。在 $\mathrm{Li}_{4} \mathrm{Ti}_{5} \mathrm{O}_{12}$ 晶胞中, 3 个 $\mathrm{Li}$ 占据 $8 \mathrm{a}$ 位, $\mathrm{Ti}$ 和剩余的 1 个 $\mathrm{Li}$ 随机占据 $16 \mathrm{~d}$ 位, 可 以写为 $\mathrm{Li}(8 \mathrm{a})[\mathrm{Li1} / 3 \mathrm{Ti} 5 / 3](16 \mathrm{~d}) \mathrm{O} 4(32 \mathrm{e})$ 。放电时, 要 嵌入 3 个 $\mathrm{Li}$ 到尖晶石结构的 $16 \mathrm{c}$ 位置, 原位于 $8 \mathrm{a}$ 位置 的 3 个 $\mathrm{Li}$ 由于静电排斥作用也转移到 $16 \mathrm{c}$ 位置, 从 $\mathrm{Li}_{4} \mathrm{Ti}_{5} \mathrm{O}_{12}$ 到 $\mathrm{Li}_{7} \mathrm{Ti}_{5} \mathrm{O}_{12}$ (可以写成 $\mathrm{Li}_{2(16 c)}\left[\mathrm{Li}_{1 / 3} \mathrm{Ti}_{5 / 3}\right]_{(16 \mathrm{c})} \mathrm{O}_{4(32 \mathrm{e})}$ ) 结构 ${ }^{[6]}$ (如图 1 所示), 晶格常数由 $0.83595 \mathrm{~nm}$ 变化到 $0.83538 \mathrm{~nm}$, 对应晶胞体积变化仅 $0.2 \%^{[7-9]}$ 。因此可 以说 $\mathrm{Li}_{4} \mathrm{Ti}_{5} \mathrm{O}_{12}$ 是一种 “零应变”材料, 具有优异的循 环稳定性。另外, $\mathrm{Li}_{4} \mathrm{Ti}_{5} \mathrm{O}_{12}$ 有比较高的电位( $1.55 \mathrm{~V} v \mathrm{~s}$. $\mathrm{Li} / \mathrm{Li}^{+}$, 碳 $0.1 \sim 0.2 \mathrm{~V}$ vs. $\mathrm{Li} / \mathrm{Li}^{+}$), 不易析出枝晶, 避 免了电池短路, 在充放电过程中安全性比较高 ${ }^{[10]}$ 。

$\mathrm{Li}_{4} \mathrm{Ti}_{5} \mathrm{O}_{12}$ 的脱嵌锂(充放电)过程可以由核壳结 构模型来描述 ${ }^{[11-12]}$ 。如图 2 所示。当锂离子嵌入 (放 电)时, 尖晶石结构的 $\mathrm{Li}_{4} \mathrm{Ti}_{5} \mathrm{O}_{12}$ 表面颗粒被还原并 转化成岩盐结构的 $\mathrm{Li}_{4} \mathrm{Ti}_{5} \mathrm{O}_{12}$ 。这个过程中形成的岩 盐结构的壳随着锂离子嵌入深度的增加而变厚, 同 时尖晶石结构的核心在逐渐缩小。放电结束后, 整 个颗粒变成岩盐结构。相反, 充电过程中岩盐结构 中的锂离子逐渐脱出进入尖晶石相, 完成脱锂过程。

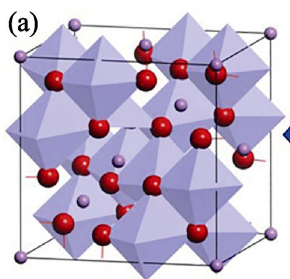

$\mathrm{Li}_{4} \mathrm{Ti}_{5} \mathrm{O}_{12}$

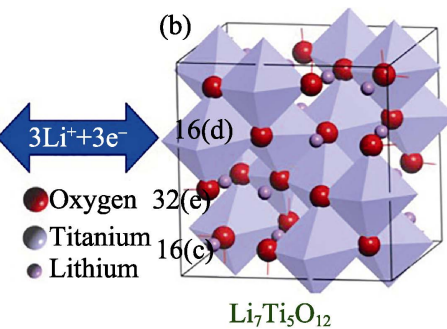

$\mathrm{Li}_{7} \mathrm{Ti}_{5} \mathrm{O}_{12}$
图 1 尖晶石型 $\mathrm{Li}_{4} \mathrm{Ti}_{5} \mathrm{O}_{12}$ 与岩盐型 $\mathrm{Li}_{7} \mathrm{Ti}_{5} \mathrm{O}_{12}$ 的结构 ${ }^{[6]}$

Fig. 1 (a) $\mathrm{Li}_{4} \mathrm{Ti}_{5} \mathrm{O}_{12}$ spinel structure type and (b) $\mathrm{Li}_{7} \mathrm{Ti}_{5} \mathrm{O}_{12}$ rock salt structure type ${ }^{[6]}$

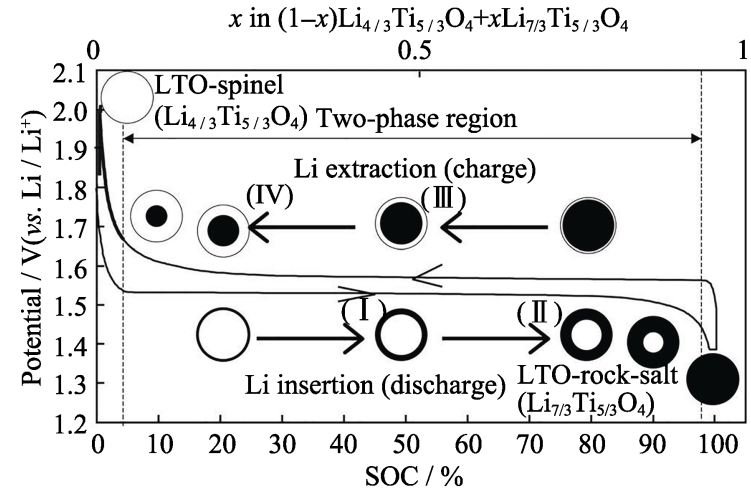

图 $2 \mathrm{Li}_{4} \mathrm{Ti}_{5} \mathrm{O}_{12}$ 脱嵌锂过程示意图 ${ }^{[12]}$

Fig. 2 Illustration of lithium insertion and extraction process of $\mathrm{Li}_{4} \mathrm{Ti}_{5} \mathrm{O}_{12}$ particle ${ }^{[12]}$

\section{2 制备方法}

制备 $\mathrm{Li}_{4} \mathrm{Ti}_{5} \mathrm{O}_{12}$ 的方法主要有固相法、溶胶一凝胶 法、水热法、喷雾热解法、熔盐法、微波法和静电 纺丝法等。不同的制备方法对合成材料的形貌和性 能影响非常大，同一物料采用不同工艺制备，会得 到不同形貌结构、不同粒度、不同电化学性能的产 品; 同时即使同一种制备工艺合成材料的性能又受 锂源和钛源的配比、反应温度、反应时间、反应气 体气氛环境等诸多因素的影响。

\section{1 固相法}

固相法是最传统的合成方法, 工艺简单, 是目 前合成很多材料的首选方法。 $\mathrm{Li}_{4} \mathrm{Ti}_{5} \mathrm{O}_{12}$ 材料通常以 $\mathrm{LiOH} \cdot \mathrm{H}_{2} \mathrm{O} 、 \mathrm{Li}_{2} \mathrm{CO}_{3} 、 \mathrm{TiO}_{2}$ 为原料, 通过高温 $(800 \sim$ $\left.1000^{\circ} \mathrm{C}\right)$ 、长时间 $(12 \mathrm{~h}$ 以上 $)$ 的煅烧形成。原料的配 比 $^{[13]}$ 、反应温度 ${ }^{[14]}$ 、反应时间 ${ }^{[15]}$ 和混料方式 ${ }^{[16-17]}$ 等合成条件都是直接影响产物 $\mathrm{Li}_{4} \mathrm{Ti}_{5} \mathrm{O}_{12}$ 的结构、形 貌和性能的重要因素。Han 等 ${ }^{[18]}$ 对高能球磨和普通 球磨以及磨球的尺寸对合成 $\mathrm{Li}_{4} \mathrm{Ti}_{5} \mathrm{O}_{12}$ 的影响进行了 深入的研究。高能球磨能够为原料提供足够的机械 活化能，更有利于单一项的合成，抑制金红石 $\mathrm{TiO}_{2}$ 的 形成, 并且能够缩短固相反应的扩散路径。当磨球 尺寸为 $0.1 \mathrm{~mm}$ 时, 制备的 $\mathrm{Li}_{4} \mathrm{Ti}_{5} \mathrm{O}_{12}$ 粒径为 $175 \mathrm{~nm}$, 拥 有更为出色的电化学性能, 放电比容量为 $174 \mathrm{mAh} / \mathrm{g}$ 。

\section{2 溶胶一凝胶法}

溶胶-凝胶法一般采用柠檬酸、草酸、丙稀酸等 作为螯合剂, 以钛酸四丁酯作为钛源, 乙酸锂、氢氧 化锂等作为锂源，水、乙醇等作为分散系，在酸的作 用下发生氧化反应 ${ }^{[19-20]}$ 。溶胶-凝胶法具有合成温度 低, 时间短, 产物粒度小、分布窄、均一性好、比表 面积大等优点 ${ }^{[21]}$ 。Liu 等 ${ }^{[22]}$ 通过两相界面反应(环己烷/ 水) 溶胶一凝胶法合成了粒径为 $100 \mathrm{~nm}$ 的 $\mathrm{Li}_{4} \mathrm{Ti}_{5} \mathrm{O}_{12}$ 
负极材料, 该材料在 $10 C$ 和 $20 C$ 倍率下循环 50 次, 放电比容量分别保持在 $150 \mathrm{mAh} / \mathrm{g}$ 和 $126.6 \mathrm{mAh} / \mathrm{g}$ 。

\section{3 水热法}

水热法也是一种常见的湿法合成方法之一, 是 将锂源和钛源置于高压釜中, 在一定温度和压力下, 在水、水溶液或蒸汽等流体中发生反应, 然后进一 步袐烧结晶 $\left(500^{\circ} \mathrm{C}\right.$ 以上)。Zhu 等 ${ }^{[23]}$ 以廉价的 $\mathrm{H}_{2} \mathrm{TiO}_{3}$ 做原料采用水热法制备了锯齿形纳米片组成的中空 分层 $\mathrm{Li}_{4} \mathrm{Ti}_{5} \mathrm{O}_{12}$ 。这种制备工艺的产量高达 $120 \mathrm{~g} / \mathrm{L}$, 并 且拥有杰出的比容量, 在 $30 C$ 倍率下, 具有 $108 \mathrm{mAh} / \mathrm{g}$ 的放电比容量, 循环 1000 次后, 放电比容量仍可保 持在 $94 \%$ 。

\section{4 其他方法}

此外, 除了上述三种方法, 许多研究者还采用

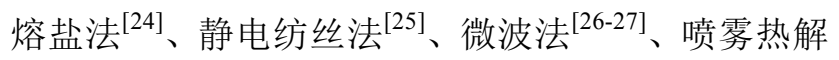
法 ${ }^{[28]}$ 等制备了性能优异的 $\mathrm{Li}_{4} \mathrm{Ti}_{5} \mathrm{O}_{12}$ 材料。

\section{$3 \quad \mathbf{L i}_{4} \mathrm{Ti}_{5} \mathrm{O}_{12}$ 材料的改性}

由于 $\mathrm{Ti}$ 原子 $3 \mathrm{~d}$ 电子层电子缺失, $\mathrm{Li}_{4} \mathrm{Ti}_{5} \mathrm{O}_{12}$ 的导 电性很差, 其电子和离子导电率分别为 $10^{-13} \mathrm{~S} / \mathrm{cm}$ 和 $10^{-9} \sim 10^{-13} \mathrm{~cm}^{2} / \mathrm{S}$, 这种绝缘属性严重影响了其在 大电流充放电条件下的倍率性能。 $\mathrm{Li}_{4} \mathrm{Ti}_{5} \mathrm{O}_{12}$ 充电过 程主要分为三步, (1) 电解液中的 $\mathrm{Li}$ 离子扩散; (2) $\mathrm{Li}_{4} \mathrm{Ti}_{5} \mathrm{O}_{12}$ 与电解液之间发生电荷转移; (3) $\mathrm{Li}$ 离子 嵌入固体 $\mathrm{Li}_{4} \mathrm{Ti}_{5} \mathrm{O}_{12}$ 中。因此, 要提高 $\mathrm{Li}_{4} \mathrm{Ti}_{5} \mathrm{O}_{12}$ 的倍 率性能, 可以通过两种方法, 一是通过离子掺杂或 表面改性的方法来提高离子扩散速率和电荷转移; 另一种方法是通过纳米化来缩短 $\mathrm{Li}$ 离子扩散的路 径 $^{[8]}$ 。

\section{1 离子掺杂}

离子掺杂是提高 $\mathrm{Li}_{4} \mathrm{Ti}_{5} \mathrm{O}_{12}$ 材料性能的有效方法 之一。对 $\mathrm{Li}_{4} \mathrm{Ti}_{5} \mathrm{O}_{12}$ 进行离子掺杂主要有两个目的, 一是降低其电极电位, 提高电池比容量; 二是提高 其导电性, 降低电阻和极化。 $\mathrm{Li}_{4} \mathrm{Ti}_{5} \mathrm{O}_{12}$ 的离子掺杂 是指在 $\mathrm{Li}^{+}$的 $8 \mathrm{a}$ 位置或者 $\mathrm{Ti}^{4+}$ 的 $16 \mathrm{~d}$ 位置引入高价 阳离子, 或者在 $\mathrm{O}^{2-}$ 的 $32 \mathrm{e}$ 位置引入低价阴离子, 促 使部分 $\mathrm{Ti}^{4+}$ 转变为 $\mathrm{Ti}^{3+}$, 以产生电荷补偿的混合物 $\left(\mathrm{Ti}^{3+} / \mathrm{Ti}^{4+}\right)$, 并增加电子浓度。同时, 掺杂会产生晶 格扭曲, 在晶格中产生新的缺陷或空隙, 因此对离 子传递的阻力发生变化, 锂离子扩散系数也随之改 变。掺杂能够提高晶格电导率和锂离子扩散系数, 提高大倍率放电性能以及循环稳定性 ${ }^{[29]}$ 。多种阴阳 离子如 $\mathrm{Na}^{+} 、 \mathrm{Mg}^{2+} 、 \mathrm{Ca}^{2+} 、 \mathrm{Sc}^{3+} 、 \mathrm{Cr}^{3+} 、 \mathrm{Cu}^{2+} 、 \mathrm{Zn}^{2+} 、 \mathrm{Sr}^{2+} 、$ $\mathrm{Sn}^{2+} 、 \mathrm{La}^{3+} 、 \mathrm{Al}^{3+}, \mathrm{V}^{5+}, \mathrm{Zr}^{4+} 、 \mathrm{Ce}^{4+}, \mathrm{Gd}^{3+} 、 \mathrm{Ta}^{5+}, \mathrm{W}^{6+} 、$ $\mathrm{Sb} 5+, \mathrm{Nd}^{3+} 、 \mathrm{Ni}^{2+} 、 \mathrm{Mn}^{4+} 、 \mathrm{Fe}^{3+} 、 \mathrm{Zn}^{2+} 、 \mathrm{Nb}^{5}+\mathrm{Ru}^{4+} 、$
$\mathrm{Bi}^{3+} 、 \mathrm{Mo}^{6+} 、 \mathrm{~F}-、 \mathrm{Cl}^{-} 、 \mathrm{Br}^{-}$等取代 $\mathrm{Li}^{+} 、 \mathrm{Ti}^{4+} 、 \mathrm{O}^{2-}$ 各 位点已被广泛研究, 并且系统的报道了离子价态和 分布等结构性质和导电特性。表 1 总结了一些代表 性阴阳离子掺杂改性 $\mathrm{Li}_{4} \mathrm{Ti}_{5} \mathrm{O}_{12}$ 材料的性能。

为了提高 $\mathrm{Li}_{4} \mathrm{Ti}_{5} \mathrm{O}_{12}$ 的导电性, 有的研究者研究 了两种元素 $\left(\mathrm{K}^{+}\right.$和 $\mathrm{Fe}^{3+[41]} 、 \mathrm{Mg}^{2+}$ 和 $\mathrm{F}^{-[42]} 、 \mathrm{Na}^{+}$和 $\mathrm{Zr}^{4+[43]} 、 \mathrm{Ni}^{2+}$ 和 $\mathrm{Mn}^{2+[44]}$ 共掺杂对 $\mathrm{Li}_{4} \mathrm{Ti}_{5} \mathrm{O}_{12}$ 的影响。 结果表明共掺杂能有效改善 $\mathrm{Li}_{4} \mathrm{Ti}_{5} \mathrm{O}_{12}$ 的导电性, 使 材料具有更好的倍率性能和循环稳定性。

另外, 有文献报道了尖晶石结构 $\mathrm{Li}_{4} \mathrm{Ti}_{5} \mathrm{O}_{12}$ 在不 同的电压范围下电化学性能不同, 特别是当延伸到 $0 \mathrm{~V}$ 时, 有利于拓宽电化学反应窗口, 可以发挥优 异的电化学性能。当放电电压延长到大约 $0 \mathrm{~V}$ 时, 化 合物中的所有 $\mathrm{Ti}^{4+}$ 被氧化, $\mathrm{Li}_{4} \mathrm{Ti}_{5} \mathrm{O}_{12}$ 可以提供 293 296 mAh/g 的理论能量。嵌锂时, 锂离子可以 嵌入 $\mathrm{Li}_{4} \mathrm{Ti}_{5} \mathrm{O}_{12}$ 晶体的三个位置, 即(8a),(16c)和(48f), 表明 $\mathrm{Li}^{+}$可以在低电位 $\left(<1.0 \mathrm{~V}\right.$ vs. $\left.\mathrm{Li} / \mathrm{Li}^{+}\right)$嵌入 $\mathrm{Li}_{4} \mathrm{Ti}_{5} \mathrm{O}_{12}$ 。这种低电位嵌锂过程将改善 $\mathrm{Li}_{4} \mathrm{Ti}_{5} \mathrm{O}_{12}$ 的 能量密度。放电过程中, 当电压范围在 $2.5 \sim 1.0 \mathrm{~V}$ 时, 3 个外加的 $\mathrm{Li}^{+}$嵌入到 $\mathrm{Li}_{4} \mathrm{Ti}_{5} \mathrm{O}_{12}$ 晶格的八面体 $(16 \mathrm{c})$ 位点, 同时, 最初位于四面体位点的 $\mathrm{Li}^{+}$也迁移到八 面体位置。当电压范围在 $1.0 \sim 0.01 \mathrm{~V}$ 时, 另外 2 个 $\mathrm{Li}^{+}$可插入岩盐结构 $\mathrm{Li}_{4} \mathrm{Ti}_{5} \mathrm{O}_{12}$ 中仍然存在的间隙位 置。空白的四面体(8a)位点可用于在 $1.0 \mathrm{~V}$ 之下的锂 离子嵌入, 从而在低电位下增强可逆容量。表 2 总 结了低电位下一些代表性阳离子掺杂改性 $\mathrm{Li}_{4} \mathrm{Ti}_{5} \mathrm{O}_{12}$ 材料的性能。

尖晶石型 $\mathrm{Li}_{4} \mathrm{Ti}_{5} \mathrm{O}_{12}$ 由于八面体位置的空缺, 为 $\mathrm{Li}^{+}$良导体。同时, 其中的 $\mathrm{Ti}$ 为最高的化合价 +4 价, 使其成为电子的不良导体。相反, 岩盐结构的 $\mathrm{Li}_{4} \mathrm{Ti}_{5} \mathrm{O}_{12}$ 中的 $\mathrm{Ti}$ 平均价态为 +3.4 价, 是一种电子的 良导体, 但随着电位降低, 嵌锂深度的增加, 由于 其 $16 \mathrm{c}$ 和 $8 \mathrm{a}$ 位点均被 $\mathrm{Li}^{+}$占据, 导致 $\mathrm{Li}^{+}$的扩散路径 受阻, $\mathrm{Li}^{+}$扩散系数的降低, 对电化学反应产生不利 的影响。同时试验用的有机电解液在 $1.0 \sim 0.5 \mathrm{~V}$ 之间 有很强的还原性, 会在电极表面还原分解形成 SEI 膜 ${ }^{[55}$, 引起电池内阻增加, 加剧电极极化, 缩短充 放电平台。

\section{2 碳材料改性}

通过导电性高、结构多样的碳材料改善 $\mathrm{Li}_{4} \mathrm{Ti}_{5} \mathrm{O}_{12}$ 材料的电化学性能是一种有效的方法。在 $\mathrm{Li}_{4} \mathrm{Ti}_{5} \mathrm{O}_{12}$ 材料中添加不同形式的碳(如无定形碳、碳纳米管、 石墨烯等), 能够促进相互连接的 $\mathrm{Li}_{4} \mathrm{Ti}_{5} \mathrm{O}_{12}$ 颗粒之 间的电子传输, 从而提高倍率性能、储锂能力和容 量保持率。 
表 1 代表性阴阳离子掺杂 $\mathrm{Li}_{4} \mathrm{Ti}_{5} \mathrm{O}_{12}$ 的 $\mathrm{Li}, \mathrm{Ti}, \mathrm{O}$ 各位点对性能的影响

Table 1 Summary of representative LTO doped with various cations/anions in the $\mathrm{Li}$, $\mathrm{Ti}$ and $\mathrm{O}$ sites

\begin{tabular}{|c|c|c|c|c|c|c|c|}
\hline Ion & $\begin{array}{l}\text { Radius } \\
\text { /nm }\end{array}$ & $\begin{array}{c}\text { Doping } \\
\text { content } \\
\text { molar ratio }\end{array}$ & Size/nm & $\begin{array}{l}\text { Initial discharge } \\
\text { capacity } /\left(\mathrm{mAh}^{-1} \mathrm{~g}^{-1}\right)\end{array}$ & $\begin{array}{l}\text { Cycle performance/ } \\
\left(\mathrm{mAh}^{-1} \mathrm{~g}^{-1}\right)\end{array}$ & Method & Ref. \\
\hline \multicolumn{8}{|c|}{ Cation doping in the Li sites(radius $0.076 \mathrm{~nm}$ ) } \\
\hline $\mathrm{Mg}^{2+}$ & 0.0720 & 0.20 & $100-200$ & $190.0(1 C)^{\mathrm{a}}$ & $179.0(1 C, 100)^{\mathrm{b}} ; 150.0(5 C, 100)$ & Solid state reaction & [30] \\
\hline $\mathrm{Ca}^{2+}$ & 0.1000 & 0.10 & $1000-2000$ & $169.7(0.5 C)$ & $162.4(1 C, 100) ; 148.8(5 C, 100)$ & Solid state reaction & {$[31]$} \\
\hline $\mathrm{Sc}^{3+}$ & 0.0745 & 0.05 & 200 & $174.0(1 C) ; 94.0(40 C)$ & $94.0(40 C, 50)$ & Sol-Gel & {$[32]$} \\
\hline $\mathrm{Cu}^{2+}$ & 0.0730 & 0.05 & $200-600$ & $158.0(0.1 C)$ & $143.8(0.1 C, 150)$ & Sol-Gel & [33] \\
\hline $\mathrm{La}^{3+}$ & 0.1032 & 0.06 & 24.5 & $169.0(0.1 C)$ & $153.4(1 C, 10) ; 146.9(5 C, 10)$ & Liquid method & [34] \\
\hline \multicolumn{8}{|c|}{ Cation doping in the Ti sites (radius $\mathrm{Ti}^{3+} 0.067 \mathrm{~nm}, \mathrm{Ti}^{4+} 0.0605 \mathrm{~nm}$ ) } \\
\hline $\mathrm{Al}^{3+}$ & 0.0535 & 0.15 & $50-200$ & $216.0(1 C) ; 163.0(10 C)$ & $180.0(5 C, 50) ; 160.0(10 C, 50)$ & $\begin{array}{l}\text { Cellulose-assisted gly- } \\
\text { cine-nitratecombustion }\end{array}$ & [35] \\
\hline $\mathrm{Zr}^{4+}$ & 0.0720 & 0.03 & 200 & $165.0(5 C) ; 152.0(10 C)$ & $142.0(5 C, 200) ; 127.0(10 C, 200)$ & Liquid method & [36] \\
\hline $\mathrm{Ce}^{4+}$ & 0.0870 & 0.10 & $<1000$ & $190.0(0.2 C) ; 40.0(2 C)$ & $140.0(2 C, 100)$ & Solid state reaction & [37] \\
\hline $\mathrm{Ta}^{5+}$ & 0.0640 & 0.05 & $500-1000$ & $193.0(0.2 C)$ & $132.0(5 C, 100)$ & Solid state reaction & [38] \\
\hline \multicolumn{8}{|c|}{ Anions doping in the $\mathrm{O}$ site (radius $0.14 \mathrm{~nm}$ ) } \\
\hline $\mathrm{Cl}^{-}$ & 0.181 & 0.2 & $3-8 \mu \mathrm{m}$ & $\begin{array}{c}148.7(0.5 C) \\
120.7(2 C)\end{array}$ & $133.8(0.5 C, 50)$ & Solid state reaction & [39] \\
\hline $\mathrm{Br}^{-}$ & 0.196 & 0.3 & $1-2 \mu \mathrm{m}$ & $174.0(0.2 C)$ & $138.0(10 C, 100) ; 104.0(210 C, 100)$ & Solid state reaction & [40] \\
\hline
\end{tabular}

Note: a: "190.0(1C)" refers to the initial discharge capacity is $190.0 \mathrm{mAh}^{-1}$ at $1 C$;

b: "179.0(1C, 100)" indicates that discharge capacity is $179.0 \mathrm{mAh}^{-1}$ after 100 cycles at $1 C$

表 2 低电位下代表性阳离子掺杂 $\mathrm{Li}_{4} \mathrm{Ti}_{5} \mathrm{O}_{12}$ 的 $\mathrm{Li}, \mathrm{Ti}, \mathrm{O}$ 各位点对性能的影响

Table 2 Summary of representative LTO doped with various cations in the $\mathrm{Li}$, Ti and $\mathrm{O}$ sites at low potential

\begin{tabular}{|c|c|c|c|c|c|c|c|}
\hline Ion & Radius/nm & $\begin{array}{l}\text { Doping content } \\
\text { molar ratio }\end{array}$ & $\begin{array}{c}\text { Size/ } \\
\mathrm{nm}\end{array}$ & $\begin{array}{c}\text { Initial discharge } \\
\text { capacity } /\left(\mathrm{mAh}^{-1}\right)\end{array}$ & $\begin{array}{l}\text { Cycle performance/ } \\
\left(\mathrm{mAh} \cdot \mathrm{g}^{-1}\right)\end{array}$ & Method & Ref. \\
\hline \multirow{4}{*}{$\mathrm{Zn}^{2+}$} & \multirow{4}{*}{0.074} & \multirow{4}{*}{0.20} & \multirow{4}{*}{$1000-2000$} & $(0-2.5 \mathrm{~V})$ & $216.4(0.5 C, 30)$ & \multirow{4}{*}{$\begin{array}{l}\text { Solid state } \\
\text { reaction }\end{array}$} & \multirow{4}{*}{ [6] } \\
\hline & & & & $271.6(0.5 C)^{\mathrm{a}}$ & $198.0(3 C, 100)$ & & \\
\hline & & & & $223.0(3 C)$ & $186.0(5 C, 200)$ & & \\
\hline & & & & $206.0(5 C)$ & & & \\
\hline \multirow{2}{*}{$\mathrm{Nb}^{5+}$} & \multirow{2}{*}{0.064} & \multirow{2}{*}{0.05} & & $(0-2.5 \mathrm{~V})$ & $231.0(0.12 C, 100)$ & \multirow{2}{*}{ Sol-Gel } & \multirow{2}{*}{ [46] } \\
\hline & & & & $351.0(0.12 C)$ & & & \\
\hline \multirow[b]{2}{*}{$\mathrm{Ru}^{4+}$} & \multirow[b]{2}{*}{0.062} & \multirow[b]{2}{*}{0.05} & \multirow[b]{2}{*}{$100-200$} & $(0.01-2.5 \mathrm{~V})$ & $259.0(3 C, 100)$ & \multirow{2}{*}{$\begin{array}{l}\text { Reverse mi- } \\
\text { croemulsion } \\
\text { method }\end{array}$} & \multirow[b]{2}{*}{ [47] } \\
\hline & & & & $274.0(3 C)$ & $131.0(60 C, 100)$ & & \\
\hline \multirow{2}{*}{$\mathrm{Bi}^{+3+}$} & \multirow{2}{*}{0.103} & \multirow{2}{*}{0.10} & \multirow{2}{*}{$500-1000$} & $(0.01-2.5 \mathrm{~V})$ & $203.0(1 C, 50)$ & \multirow{2}{*}{$\begin{array}{l}\text { Solid state } \\
\text { reaction }\end{array}$} & \multirow{2}{*}{ [48] } \\
\hline & & & & $214.0(1 C)$ & & & \\
\hline
\end{tabular}

Note: a: "(0-2.5 V)271.6(0.5C)" refers to the initial discharge capacity is $271.6 \mathrm{mAh}^{-1}$ at $0.5 C$ from $0 \mathrm{~V}$ to $2.5 \mathrm{~V}$

\subsection{1 表面碳包覆}

碳包覆是目前最为常见的 $\mathrm{Li}_{4} \mathrm{Ti}_{5} \mathrm{O}_{12}$ 改性方法, 碳包覆不仅可以改善表面电导率，而且可以改善 $\mathrm{Li}_{4} \mathrm{Ti}_{5} \mathrm{O}_{12}$ 材料和电解质之间的接触, 减小电池阻抗 和电荷转移的阻力。加热使含碳物质分解, 在 $\mathrm{Li}_{4} \mathrm{Ti}_{5} \mathrm{O}_{12}$ 表面形成厚度均匀的导电碳层, 增强了 $\mathrm{Li}_{4} \mathrm{Ti}_{5} \mathrm{O}_{12}$ 的导电性，从而改善其电化学性能。同时 添加的碳源在煅烧过程中还能够抑制颗粒在高温热 处理过程中的长大，从而减小最终颗粒的粒径。碳 含量高有助于提高表面电导率，但也会产生较厚的
碳包覆涂层，这样会限制锂离子的扩散，只有优化 后均匀的碳包覆涂层可以提供良好的导电性和快速 锂离子传输通道。表 3 总结了不同碳源、含量、厚 度对碳包覆 $\mathrm{Li}_{4} \mathrm{Ti}_{5} \mathrm{O}_{12}$ 性能的影响。

\subsection{2 石墨烯改性 $\mathrm{Li}_{4} \mathrm{Ti}_{5} \mathrm{O}_{12}$ 复合材料}

碳材料除可作为包覆层外, 还可利用自身的结 构优势，与 $\mathrm{Li}_{4} \mathrm{Ti}_{5} \mathrm{O}_{12}$ 构成具有特殊形貌结构的复合 材料。石墨烯是具有蜂窝结构的碳原子的二维大分 子片，具有很好的电子导电率和柔韧性，是理想的 导电添加剂 ${ }^{[54]}$ 。 $\mathrm{Li}$ 等 ${ }^{[55]}$ 将钛酸四丁酯和石墨烯分散 
表 3 不同碳源、含量、厚度对碳包覆 $\mathbf{L i}_{4} \mathbf{T i}_{5} \mathbf{O}_{12}$ 的性能影响

Table 3 The effects of the carbon source, carbon content, thickness, and graphitization on the electrochemical performance of the carbon coated $\mathrm{Li}_{4} \mathrm{Ti}_{5} \mathrm{O}_{12}$

\begin{tabular}{|c|c|c|c|c|c|c|}
\hline Carbon source & $\begin{array}{c}\text { Carbon } \\
\text { content } / \mathrm{wt} \%\end{array}$ & $\begin{array}{c}\text { Thickness/ } \\
\text { nm }\end{array}$ & $\begin{array}{l}\text { Initial discharge } \\
\text { capacity } /\left(\mathrm{mAh}^{-1} \mathrm{~g}^{-1}\right)\end{array}$ & $\begin{array}{c}\text { Cycle performance/ } \\
\left(\mathrm{mAh} \cdot \mathrm{g}^{-1}\right)\end{array}$ & Method & Ref. \\
\hline $\mathrm{LPAN}+\mathrm{CB}$ & 3.64 & $3-5$ & $166.2(10 C)$ & $141.9(10 C, 200)$ & Solid state reaction & [49] \\
\hline PEDOT & 10.00 & 10 & $168.7(1 C)$ & $167.9(1 C, 100)$ & Hydrothermal reaction & {$[50]$} \\
\hline Sucrose & 8.60 & $2-4$ & $\begin{array}{l}156.7(40 C) ; \\
142.1(60 C) ; \\
132.8(80 C)\end{array}$ & $\begin{array}{c}114.2(40 C, 200) \\
98.1(60 C, 200) \\
82.7(80 C, 200)\end{array}$ & $\begin{array}{l}\text { One-step } \\
\text { liquid } \\
\text { process }\end{array}$ & {$[51]$} \\
\hline Citric acid & 1.32 & $2-3$ & $\begin{array}{c}165.7(1 C) ; \\
161.7(5 C) ; \\
153.9(10 C) ; \\
147.9(20 C)\end{array}$ & $144.9(20 C, 50)$ & Sol-Gel & {$[52]$} \\
\hline Glucose & 8.96 & 8 & $170.9(0.5 C)$ & $155.6(1 C, 100)$ & $\begin{array}{l}\text { Hydrothermal-solid } \\
\text { state reaction }\end{array}$ & {$[53]$} \\
\hline
\end{tabular}

在叔丁醇中，通过微波照射加热到回流状态，随后加 入醋酸锂制备前驱体 $p-\mathrm{Li}_{4} \mathrm{Ti}_{5} \mathrm{O}_{12} / \mathrm{G}$ 。前驱体形态均匀, 尺寸超小，通过裆烧形成 $\mathrm{Li}_{4} \mathrm{Ti}_{5} \mathrm{O}_{12} @ \mathrm{G}, \mathrm{Li}_{4} \mathrm{Ti}_{5} \mathrm{O}_{12} @ \mathrm{G}$ 进一步与石墨烯片混合制备 $\mathrm{G} @ \mathrm{LI}_{4} \mathrm{TI}_{5} \mathrm{O}_{12} @ \mathrm{G}$ (图 3)。所制备的 $\mathrm{G} @ \mathrm{Li}_{4} \mathrm{Ti}_{5} \mathrm{O}_{12} @ \mathrm{G}$ 具有三维结构和分层 多空分布。这种独特的结构提供了巨大的振实密度、 快速电子转移和电解质运输的通道。该材料在 $5 \mathrm{C}$ 倍率下的初始放电容量为 $168.6 \mathrm{mAh} / \mathrm{g}, 1000$ 次循环 后容量仍然保持在 $163.8 \mathrm{mAh} / \mathrm{g}$, 容量保持率达到 $97.2 \%$ 。Yang 等 ${ }^{[56]}$ 使用冷冻干燥辅助微波照射的方 法将包裹在碳壳内的 $\mathrm{Li}_{4} \mathrm{Ti}_{5} \mathrm{O}_{12}$ 颗粒针定到石墨烯纳 米片(图 4)。这种结构的设计通过连接石墨烯纳米片 和碳层形成一个可以导电的三维网络, 有利于电子 和锂离子的传递，均匀的碳涂层和石墨烯纳米片的 包覆，改善了每个 $\mathrm{Li}_{4} \mathrm{Ti}_{5} \mathrm{O}_{12}$ 颗粒的电子传导性。该 材料在 $1 C 、 2 C 、 5 C 、 10 C$ 和 $20 C$ 倍率下比容量分别 为 $171.5 、 168.2 、 160.1 、 151.7$ 和 $136.4 \mathrm{mAh} / \mathrm{g}$ 。20C 倍 率 20 次循环后容量保持率仍为 $99.6 \%$ 。 Kim 等 ${ }^{[57]}$ 通过彻底清洗和透析制备了高质量、含最少杂质的 石墨烯氧化物，这使得每个单层石墨烯都能够成功
的包裹每个 $\mathrm{Li}_{4} \mathrm{Ti}_{5} \mathrm{O}_{12}$ 颗粒(图 5)。单层石墨烯包覆 $\mathrm{Li}_{4} \mathrm{Ti}_{5} \mathrm{O}_{12}$ 粒度 $200 \mathrm{~nm}$, 表现出优异的性能, 即使在 $30 C$ 倍率下, 脱嵌锂比容量仍可达到 $130 \mathrm{mAh} / \mathrm{g}$; $10 C$ 倍率下循环 200 次, 容量保持在 $151 \mathrm{mAh} / \mathrm{g}$ 。

\section{3 形貌改进}

$\mathrm{Li}_{4} \mathrm{Ti}_{5} \mathrm{O}_{12}$ 结构的纳米化，或者制备出一维、二 维或三维结构的 $\mathrm{Li}_{4} \mathrm{Ti}_{5} \mathrm{O}_{12}$, 可以有效改善其电化学 性能。纳米结构能够缩短电子和锂离子在 $\mathrm{Li}_{4} \mathrm{Ti}_{5} \mathrm{O}_{12}$ 颗粒内的运输路径, 并且提供了更大的电极/电解液 的接触面积, 有利于改善锂离子嵌入的动力学 ${ }^{[58]}$ 。 Luo 等 ${ }^{[59]}$ 通过水热法以葡萄糖作为碳源包覆锐钛矿 $\mathrm{TiO}_{2}$, 在 $\mathrm{LiOH}$ 溶液中生成 $\mathrm{Li}_{4} \mathrm{Ti}_{5} \mathrm{O}_{12} / \mathrm{C}$ 纳米棒。与 纯 $\mathrm{Li}_{4} \mathrm{Ti}_{5} \mathrm{O}_{12}$ 相比, 这种纳米棒结构(图 6)具有丰富 的分层孔隙, 比表面积为 $107.8 \mathrm{~m}^{2} / \mathrm{g}$, 增加了锂离子 脱嵌的表面积, 加速了锂离子与电子的传递。在 $0.2 \mathrm{C}$ 倍率下, 首次放电比容量为 $168.4 \mathrm{mAh} / \mathrm{g}$, 库伦 效率 $95 \%$ 。 $\mathrm{Liu}$ 等 $^{[60]}$ 设计了一种具有高导电性的自 支撑 $\mathrm{Li}_{4} \mathrm{Ti}_{5} \mathrm{O}_{12}-\mathrm{C}$ 纳米管阵列结构(图 7)。 $\mathrm{Li}_{4} \mathrm{Ti}_{5} \mathrm{O}_{12}$ 纳米管阵列通过简单的基于模板溶液法直接生长在不

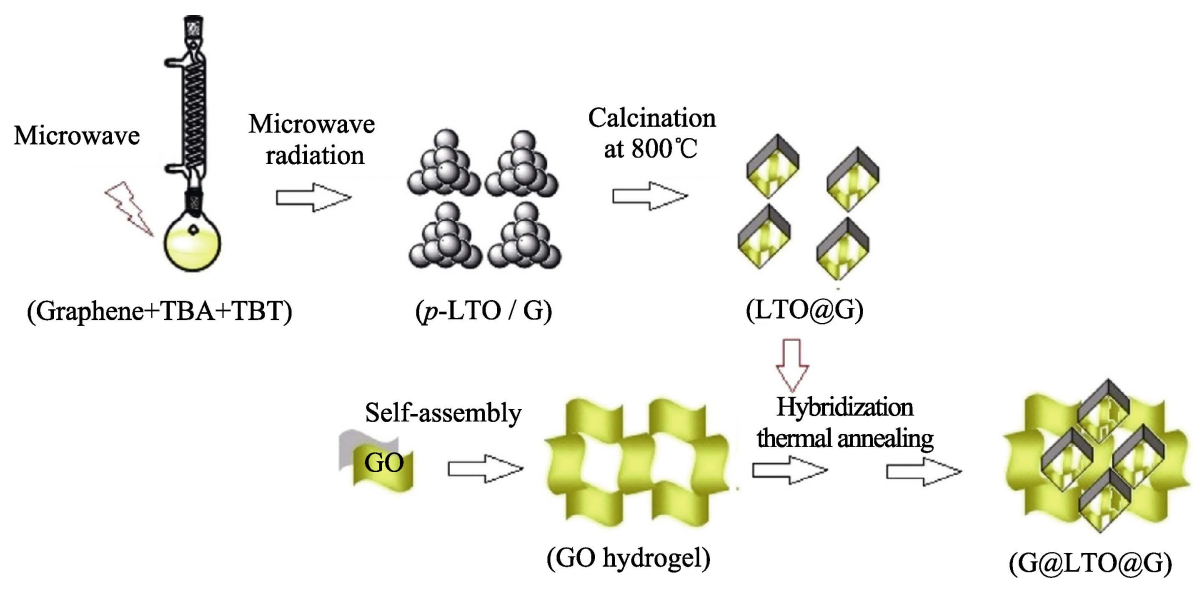

图 3 制备 $\mathrm{G} @ \mathrm{Li}_{4} \mathrm{Ti}_{5} \mathrm{O}_{12} @ \mathrm{G}$ 过程示意图 ${ }^{[55]}$

Fig.3 The procedure for the preparation of G@LTO@G $\mathrm{G}^{[55]}$ 


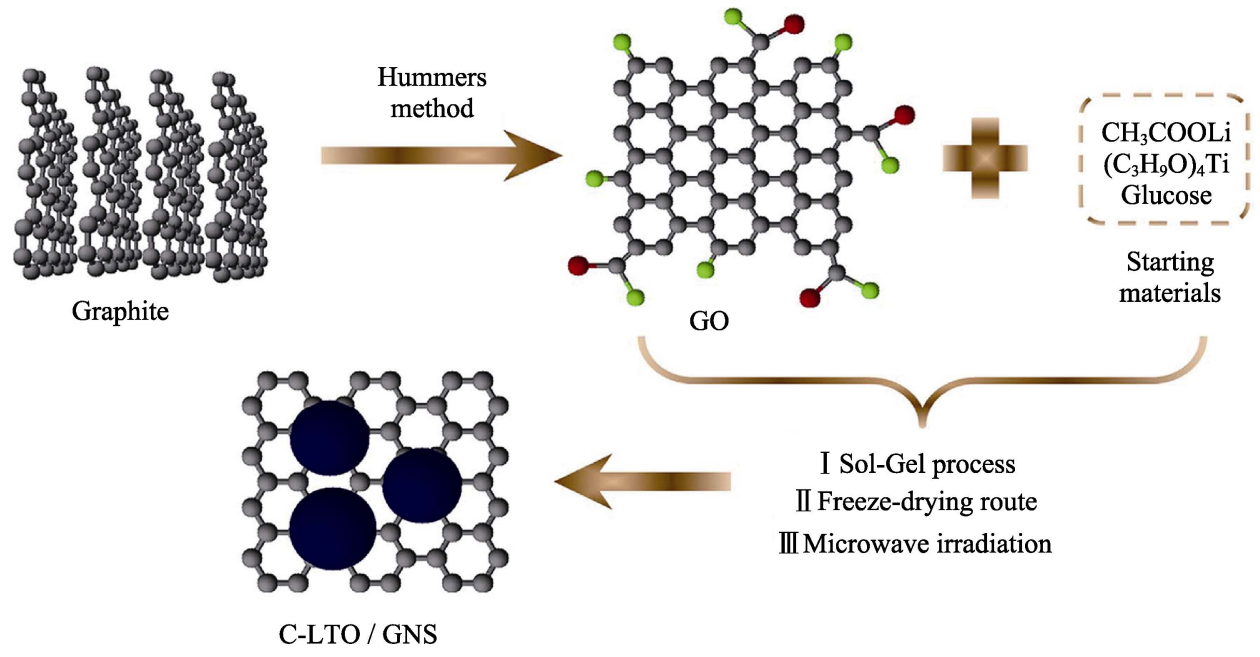

图 4 制备 $\mathrm{C}-\mathrm{Li}_{4} \mathrm{Ti}_{5} \mathrm{O}_{12} / \mathrm{GNS}$ 过程示意图 ${ }^{[56]}$

Fig. 4 Schematic illustration for the fabrication of C-LTO/GNS composite ${ }^{[56]}$

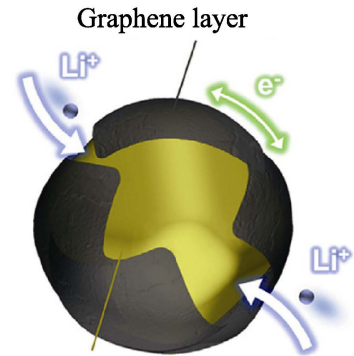

LTO grain

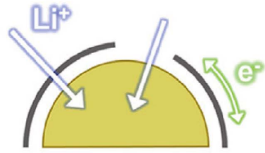

$0.5 \mathrm{wt} \% \mathrm{G}-\mathrm{LTO}$

(Half layer)
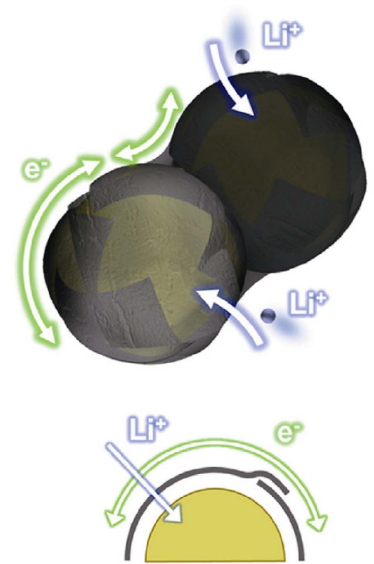

$1.8 \mathrm{wt} \% \mathrm{G}-\mathrm{LTO}$

(Single layer)

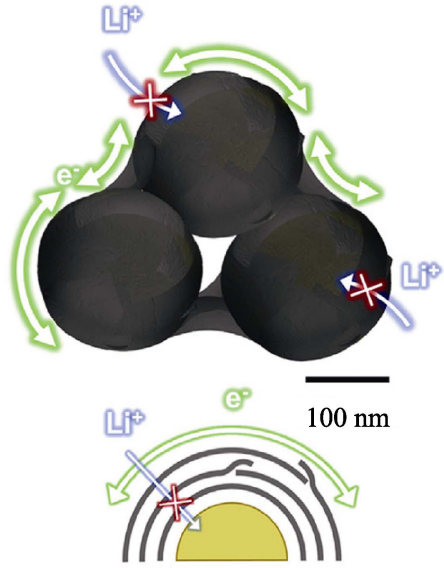

2.9wt \% G-LTO

(Three layer)

图 $5 \mathrm{Li}_{4} \mathrm{Ti}_{5} \mathrm{O}_{12}$ 表面石墨烯层数的电子传导和锂离子传输过程示意图 ${ }^{[57]}$

Fig. 5 Schematic illustration representing the electron conduction $\mathrm{Li}^{+}$and $\mathrm{Li}^{+}$transport according to the number of graphene layers on the LTO surface ${ }^{[57]}$

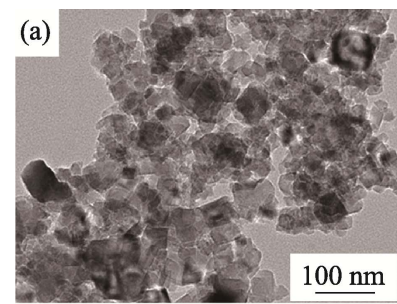

(b)

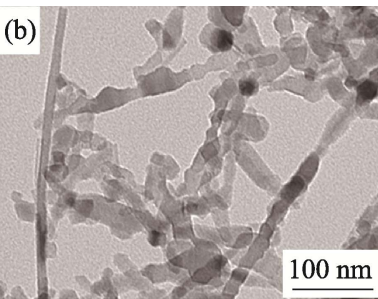

(c)

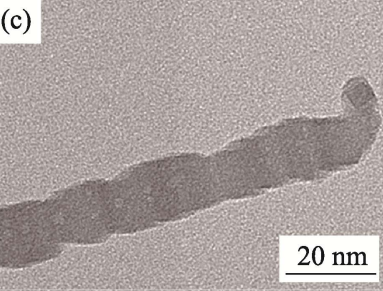

(d)

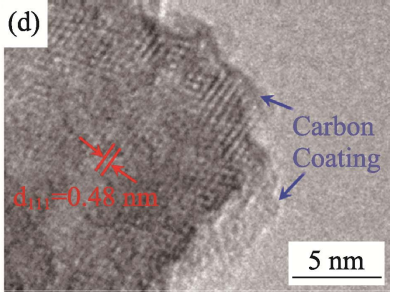

图 $6 \mathrm{Li}_{4} \mathrm{Ti}_{5} \mathrm{O}_{12}$ 的 TEM 照片 (a), $\mathrm{Li}_{4} \mathrm{Ti}_{5} \mathrm{O}_{12} / \mathrm{C}$ 纳米棒的 TEM 照片 (b, c) 和 HRTEM 照片 (d) ${ }^{[59]}$

Fig. 6 (a) TEM image of the $\mathrm{Li}_{4} \mathrm{Ti}_{5} \mathrm{O}_{12}$ nanoparticles and (b, c) TEM and (d) HRTEM images of $\mathrm{Li}_{4} \mathrm{Ti}_{5} \mathrm{O}_{12} / \mathrm{C}$ nanorods ${ }^{[59]}$
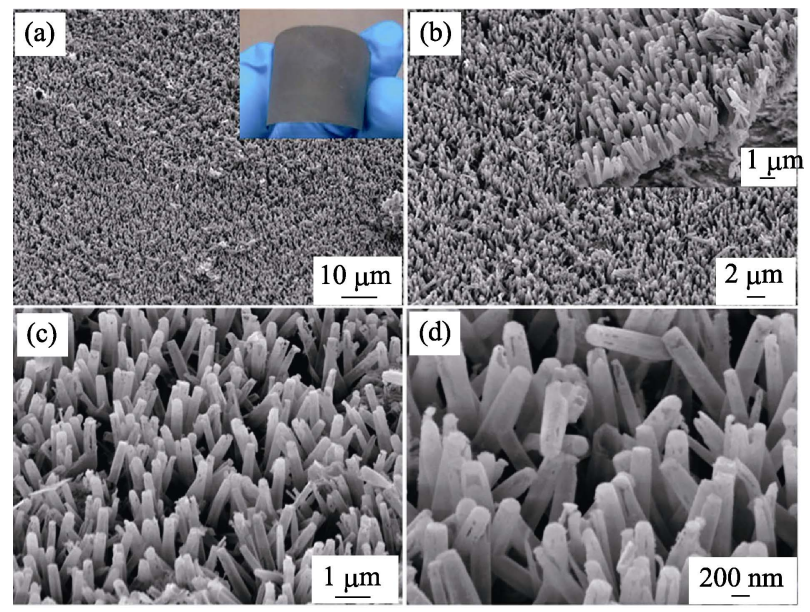

图 7 不同放大倍数的 $\mathrm{Li}_{4} \mathrm{Ti}_{5} \mathrm{O}_{12}-\mathrm{C}$ 纳米管阵列的 $\mathrm{SEM}$ 照片 ${ }^{[60]}$ Fig. 7 Different magnification SEM images of $\mathrm{Li}_{4} \mathrm{Ti}_{5} \mathrm{O}_{12}-\mathrm{C}$ nanotube arrays on stainless steel foil ${ }^{[60]}$ 
锈钢箔上, 再在其内外表面均匀涂覆碳层, 进一步 提高了导电性。该材料在 30C、60C、100C 倍率下, 可逆比容量分别为 135,105 和 $80 \mathrm{mAh} / \mathrm{g}$ 。并且在 $10 C$ 倍率下循环 500 次后, 容量保持为 $144 \mathrm{mAh} / \mathrm{g}$ 。

\section{4 展望}

尖晶石结构的 $\mathrm{Li}_{4} \mathrm{Ti}_{5} \mathrm{O}_{12}$ 具有安全性高、结构稳 定、循环性能好, $1.5 \mathrm{~V}\left(v s . \mathrm{Li} / \mathrm{Li}^{+}\right)$稳定的电压平台等 优点, 在动力储能方面备受瞩目, 成为最有潜力的下 一代锂电池负极材料之一。当前对 $\mathrm{Li}_{4} \mathrm{Ti}_{5} \mathrm{O}_{12}$ 材料的 研究仍存在不足, 需要从以下几个方面解决:

1)锂离子电池负极材料的研究重点是高比容 量、高效率、循环稳定和耐高低温环境。 $\mathrm{Li}_{4} \mathrm{Ti}_{5} \mathrm{O}_{12}$ 作为锂离子电池负极材料, 在循环稳定性和安全性 方面优势明显, 但仍无法满足锂离子蓄电池对负极 材料容量的要求(碳材料 $372 \mathrm{mAh} / \mathrm{g}$ )。因此需要通过 优化结构等方法深入研究 $\mathrm{Li}_{4} \mathrm{Ti}_{5} \mathrm{O}_{12}$ 不同形貌与尺寸 对于性能的影响, 进一步提高其能量密度。

2)针对 $\mathrm{Li}_{4} \mathrm{Ti}_{5} \mathrm{O}_{12}$ 导电率低的缺陷, 通过离子掺 杂能够改善 $\mathrm{Li}_{4} \mathrm{Ti}_{5} \mathrm{O}_{12}$ 材料的导电性, 已有很多研究, 但对于哪种掺杂离子对 $\mathrm{Li}_{4} \mathrm{Ti}_{5} \mathrm{O}_{12}$ 材料的改性最大, 其掺杂的位置、机理等有待更深入研究。尤其是共 掺杂方法, 对 $\mathrm{Li}_{4} \mathrm{Ti}_{5} \mathrm{O}_{12}$ 材料结构变化、界面特征、 共掺杂离子与基体之间的相互作用以及离子分布仍 需要进一步研究。

3)目前对 $\mathrm{Li}_{4} \mathrm{Ti}_{5} \mathrm{O}_{12}$ 材料高低温环境性能研究的 比较少, 今后的研究工作重点应放在高低温环境对 $\mathrm{Li}_{4} \mathrm{Ti}_{5} \mathrm{O}_{12}$ 电性能的影响, 加快促进 $\mathrm{Li}_{4} \mathrm{Ti}_{5} \mathrm{O}_{12}$ 材料在 工业上的应用。

\section{参考文献:}

[1] LI Z Y, DING F X, ZHAO Y G, et al. Synthesis and electrochemical performance of $\mathrm{Li}_{4} \mathrm{Ti}_{5} \mathrm{O}_{12}$ submicrospheres coated with TiN as anode materials for lithium-ion battery. Ceram. Int., 2016, 42(14): 15464-15470.

[2] HUI Y N, CAO L Y, XU Z W, et al. In situ synthesis of core-shell $\mathrm{Li}_{4} \mathrm{Ti}_{5} \mathrm{O}_{12} @$ polyaniline composites with enhanced rate performance for lithium-ion battery anodes. J. Mater. Sci. Techno., 2016, 33(3): 231-238.

[3] CHEN S, XIN Y L, ZHOU Y Y, et al. Self-supported $\mathrm{Li}_{4} \mathrm{Ti}_{5} \mathrm{O}_{12}$ nanosheet arrays for lithium ion batteries with excellent rate capability and ultralong cycle life. Energy Environ. Sci., 2014, 7(6): 1924-1930.

[4] YI T F, YANG S Y, XIE Y. Recent advances of $\mathrm{Li}_{4} \mathrm{Ti}_{5} \mathrm{O}_{12}$ as a promising next generation anode material for high power lithiumion batteries. J. Mater. Chem. A, 2015, 3(11): 5750-5777.

[5] BORGHOLS W J H, WAGEMAKER M, LAFONT U, et al. Size effects in the $\mathrm{Li}_{4+\times} \mathrm{Ti}_{5} \mathrm{O}_{12}$ spinel. J. Am. Chem. Soc., 2009, 131(49): 17786-17792.

[6] YI T F, LIU H P, ZHU Y R, et al. Improving the high rate per- formance of $\mathrm{Li}_{4} \mathrm{Ti}_{5} \mathrm{O}_{12}$ through divalent zinc substitution. J. Power Sources, 2012, 215: 258-265.

[7] WIKENING M, IWANIAK W, HEINE J, et al. Microscopic Li self-diffusion parameters in the lithiated anode material $\mathrm{Li}_{4+} \mathrm{Ti}_{5} \mathrm{O}_{12}(0<$ or $=x<$ or $=3$ ) measured by ${ }^{7} \mathrm{Li}$ solid state NMR. Phys. Chem. Chem. Phys., 2007, 9(47): 6199-6202.

[8] YANG S, WANG Q F, LU M W, et al. Synthesis of graphitized carbon, nanodiamond and graphene supported $\mathrm{Li}_{4} \mathrm{Ti}_{5} \mathrm{O}_{12}$ and comparison of their electrochemical performance as anodes for lithium ion batteries. Appl. Surf. Sci., 2016, 389: 428-437.

[9] ZHU G N, WANG Y G, XIA Y Y. Ti-based compounds as anode materials for Li-ion batteries. Energy Environ. Sci., 2012, 5(5): 6652-6667.

[10] Yang L Y, LI H Z, LIU J, et al. Effects of $\mathrm{TiO}_{2}$ phase on the performance of $\mathrm{Li}_{4} \mathrm{Ti}_{5} \mathrm{O}_{12}$ anode for lithium-ion batteries. J. Alloys Compd., 2016, 689: 812-819.

[11] MA J, WANG C, WROBLEWSKI S. Kinetic characteristics of mixed conductive electrodes for lithium ion batteries. J. Power Sources, 2007, 164(2): 849-856.

[12] TAKAMI N, HOSHINA K, INAGAKI H. Lithium diffusion in $\mathrm{Li}_{4} / 3 \mathrm{Ti}_{5} / 3 \mathrm{O}_{4}$ particles during insertion and extraction. J. Electrochem. Soc., 2011, 158(6): A725-A730.

[13] LIU W, ZHANG J, WANG Q, et al. The effects of $\mathrm{Li}_{2} \mathrm{CO}_{3}$ particle size on the properties of lithium titanate as anode material for lithium-ion batteries. Ionics, 2014, 20 (11): 1553-1560.

[14] CHEN C, SPEARS M, WONDRE F, et al. Crystal growth and superconductivity of $\mathrm{LiTi}_{2} \mathrm{O}_{4}$ and $\mathrm{Li}_{1+1 / 3} \mathrm{Ti}_{2-1 / 3} \mathrm{O}_{4}$. J. Cryst. Growth, 2003, 250(1/2): 139-145.

[15] GUERFI A, SEVIGNY S, LAGACE M, et al. Nano-particle $\mathrm{Li}_{4} \mathrm{Ti}_{5} \mathrm{O}_{12}$ spinel as electrode for electrochemical generators. J. Power Sources, 2003, 119(121): 88-94.

[16] MICHALSKA M, KRAJEWSKI M, ZIOLKOWSKA D, et al. Influence of milling time in solid-state synthesis on structure, morphology and electrochemical properties of $\mathrm{Li}_{4} \mathrm{Ti}_{5} \mathrm{O}_{12}$ of spinel structure. Powder Technol., 2014, 266(6): 372-377.

[17] SENNA M, FAVIAN M, KAVAN L, et al. Electrochemical properties of spinel $\mathrm{Li}_{4} \mathrm{Ti}_{5} \mathrm{O}_{12}$ nanoparticles prepared via a low-temperature solid route. J. Solid State Electrochem., 2016, 20(10): 2673-2683.

[18] HAN S W, JEONG J, YOON D H. Effects of high-energy milling on the solid-state synthesis of pure nano-sized $\mathrm{Li}_{4} \mathrm{Ti}_{5} \mathrm{O}_{12}$ for high power lithium battery applications. Appl. Phys. A, 2014, 114(3): 925-930.

[19] WANG Y Q, ZHAO J, QU J, et al. Investigation into the surface chemistry of $\mathrm{Li}_{4} \mathrm{Ti}_{5} \mathrm{O}_{12}$ nanoparticles for lithium ion batteries. Appl. Mater. Interfaces, 2016, 8(39): 26008-26012.

[20] QIU C X, YUAN Z Z, LIU L, et al. Sol-Gel preparation and electrochemical properties of La-doped $\mathrm{Li}_{4} \mathrm{Ti}_{5} \mathrm{O}_{12}$ anode material for lithium-ion battery. J. Solid State Electrochem., 2013, 17(3): 841847.

[21] MOHAMMADI M R, FRAY D J. Low temperature nanostructured lithium titanates: controlling the phase composition, crystal structure and surface area. J. Sol-Gel Sci. Techn., 2010, 55(1): 19-35.

[22] LIU G Y, ZHANG R X, BAO K Y, et al. Synthesis of nano$\mathrm{Li}_{4} \mathrm{Ti}_{5} \mathrm{O}_{12}$ anode material for lithium ion batteries by a biphasic interfacial reaction route. Ceram. Int., 2016, 42(9): 11468-11472.

[23] ZHU K X, GAO H Y, HU G X, et al. Scalable synthesis of hierarchical hollow $\mathrm{Li}_{4} \mathrm{Ti}_{5} \mathrm{O}_{12}$ microspheres assembled by zigzag-like nanosheets for high rate lithium-ion batteries. J. Power Sources, 2017, 340: 263-272.

[24] GUO Q J, LI S Y, WANG H, et al. Molten salt synthesis of nano-sized $\mathrm{Li}_{4} \mathrm{Ti}_{5} \mathrm{O}_{12}$ doped with $\mathrm{Fe}_{2} \mathrm{O}_{3}$ for use as anode material in the lithium ion battery. RSC Adv., 2014. 4(104): 60327-60333. 
[25] ZHAO Y G, LI J L, LI Z Y, et al. Pr-modified $\mathrm{Li}_{4} \mathrm{Ti}_{5} \mathrm{O}_{12}$ nanofibers as an anode material for lithium-ion batteries with outstanding cycling performance and rate performance. Ionics, 2017. 23(3): 597-605.

[26] YANG L H, DONG C D, GUO J. Hybrid microwave synthesis and characterization of the compounds in the Li-Ti-O system. J. Power Sources, 2008, 175(1): 575-580.

[27] LI J , JIN Y L, ZHANG X G, et al. Microwave solid-state synthesis of spinel $\mathrm{Li}_{4} \mathrm{Ti}_{5} \mathrm{O}_{12}$ nanocrystallites as anode material for lithiumion batteries. Solid State Ionics, 2007, 178(29/30): 1590-1594.

[28] JIA X L, KAN Y F, ZHU X, et al. Building flexible $\mathrm{Li}_{4} \mathrm{Ti}_{5} \mathrm{O}_{12} / \mathrm{CNT}$ lithium-ion battery anodes with superior rate performance and ultralong cycling stability. Nano Energy, 2014, 10: 344-352.

[29] WOLFENSTINE J, ALLEN J L. Electrical conductivity and charge compensation in $\mathrm{Ta}$ doped $\mathrm{Li}_{4} \mathrm{Ti}_{5} \mathrm{O}_{12}$. J. Power Sources, 2008, 180(1): 582-585.

[30] LI F Y, ZHENG M, LI J, et al. Preparation and electrochemical performance of Mg-doped $\mathrm{Li}_{4} \mathrm{Ti}_{5} \mathrm{O}_{12}$ nanoparticles as anode materials for lithium-ion batteries. Int. J. Electrochem. Sci., 2015, 10(12): 10445-10453.

[31] ZHANG Q Y, ZHANG C L, LI B, et al. Preparation and electrochemical properties of $\mathrm{Ca}$-doped $\mathrm{Li}_{4} \mathrm{Ti}_{5} \mathrm{O}_{12}$ as anode materials in lithium-ion battery materials for flexible Li-ion batteries. Electrochim. Acta, 2013, 98(16): 146-152.

[32] ZHANG Y Y, ZHANG C M, LIN Y, et al. Influence of $\mathrm{Sc}^{3+}$ doping in B-site on electrochemical performance of $\mathrm{Li}_{4} \mathrm{Ti}_{5} \mathrm{O}_{12}$ anode materials for lithium-ion battery. J. Power Sources, 2014, 250(3): $50-57$.

[33] GE Y Q, JIANG H, FU K, et al. Copper-doped $\mathrm{Li}_{4} \mathrm{Ti}_{5} \mathrm{O}_{12} /$ carbon nanofiber composites as anode for high-performance sodium-ion batteries. J. Power Sources, 2014, 272: 860-865.

[34] BAI Y J, GONG C, QI Y X, et al. Excellent long-term cycling stability of La-doped $\mathrm{Li}_{4} \mathrm{Ti}_{5} \mathrm{O}_{12}$ anode material at high current rates. $J$. Mater. Chem., 2012, 22(36): 19054-19060.

[35] CAI R, JIANG S M, YU X, et al. A novel method to enhance rate performance of an $\mathrm{Al}$-doped $\mathrm{Li}_{4} \mathrm{Ti}_{5} \mathrm{O}_{12}$ electrode by post-synthesis treatment in liquid formaldehyde at room temperature. J. Mater. Chem., 2012, 22(16): 8013-8021.

[36] CHEN Y J, MU D B, HANG R, et al. The Improvement of Discharge Capacity of Zr-doped Lithium Titanate for Lithium Ion Batteries. Matec Web Conf., 2016, 67: 06028.

[37] ZHOU T P, FENG X Y, GUO X, et al. Solid-state synthesis and electrochemical performance of Ce-doped $\mathrm{Li}_{4} \mathrm{Ti}_{5} \mathrm{O}_{12}$ anode materials for lithium-ion batteries. Electrochim. Acta, 2015, 174: 369-375.

[38] GUO M, WANG S Q, DING L X, et al. Tantalum-doped lithium titanate with enhanced performance for lithium-ion batteries. $J$. Power Sources, 2015, 283(25): 372-380.

[39] HUANG Y D, QI Y L, JIA D Z, et al. Synthesis and electrochemical properties of spinel $\mathrm{Li}_{4} \mathrm{Ti}_{5} \mathrm{O}_{12-} \mathrm{Cl}_{x}$ anode materials for lithiumion batteries. J. Solid State Electrochem., 2012, 16(5): 2011- 2016.

[40] WANG J Q, YANG Z Z, LI W H, et al. Nitridation Br-doped $\mathrm{Li}_{4} \mathrm{Ti}_{5} \mathrm{O}_{12}$ anode for high rate lithium ion batteries. J. Power Sources, 2014, 266(1): 323-331.

[41] DING K Q, ZHAO J, SUN Y Z, et al. Using potassium ferricyanide as a dopant to prepare $\mathrm{K}$ and $\mathrm{Fe}$ co-doped $\mathrm{Li}_{4} \mathrm{Ti}_{5} \mathrm{O}_{12}$. Ceram. Int., 2016, 42(16): 19187-19194.

[42] BAI X, LI W, WEI A J, et al. Preparation and electrochemical properties of $\mathrm{Mg}^{2+}$ and $\mathrm{F}^{-}$co-doped $\mathrm{Li}_{4} \mathrm{Ti}_{5} \mathrm{O}_{12}$ anode material for use in the lithium-ion batteries materials for flexible Li-ion batteries. Electrochim. Acta, 2016, 222: 1045-1055.
[43] XU P, HUANG X B, REN Y R, et al. $\mathrm{Na}^{+}$and $\mathrm{Zr}^{4+}$ co-doped $\mathrm{Li}_{4} \mathrm{Ti}_{5} \mathrm{O}_{12}$ as anode materials with superior electrochemical performance for lithium ion batteries. RSC Adv., 2016, 6(93): 90455 90461.

[44] LONG W M, WANG X Y, YANG S Y, et al. Electrochemical properties of $\mathrm{Li}_{4} \mathrm{Ti}_{5-2 x} \mathrm{Ni}_{x} \mathrm{Mn}_{x} \mathrm{O}_{12}$ compounds synthesized by Sol-Gel process. Mater. Chem. Phys., 2011, 131(1): 431-435.

[45] SHKROB I A, ZHU Y, MARIN T W, et al. Reduction of carbonate electrolytes and the formation of solid-electrolyte interface (SEI) in lithium-ion batteries. 1. spectroscopic observations of radical intermediates generated in one-electron reduction of carbonates. $J$. Phys. Chem. C, 2013, 117(38): 19255-19269.

[46] TIAN B B, XIANG H F, ZHANG L, et al. Effect of Nb-doping on electrochemical stability of $\mathrm{Li}_{4} \mathrm{Ti}_{5} \mathrm{O}_{12}$ discharged to $0 \mathrm{~V}$. J. Solid State Electrochem., 2012, 16(1): 205-211.

[47] WANG W, WANG H L, WANG S B, et al. Ru-doped $\mathrm{Li}_{4} \mathrm{Ti}_{5} \mathrm{O}_{12}$ anode materials for high rate lithium-ion batteries. J. Power Sources, 2013, 228(11): 244-249.

[48] SUBBURAJ T, PRASANNA K, KIM K J, et al. Structural and electrochemical evaluation of bismuth doped lithium titanium oxides for lithium ion batteries. J. Power Sources, 2015, 280: 23-29.

[49] HUANG P X, TANG S H, PENG H, et al. In-situ synthesis of graphitized-carbon coated $\mathrm{Li}_{4} \mathrm{Ti}_{5} \mathrm{O}_{12} / \mathrm{C}$ anode for high-rate lithium ion batteries. Mater. Sci. Forum, 2015, 814: 358-364.

[50] WANG X Y, SHEN L F, LI H S, et al. PEDOT coated $\mathrm{Li}_{4} \mathrm{Ti}_{5} \mathrm{O}_{12}$ nanorods: soft chemistry approach synthesis and their lithium storage properties. Electrochim. Acta, 2014, 129(16): 283-289.

[51] MU D B, CHEN Y J, WU B R, et al. Nano-sized $\mathrm{Li}_{4} \mathrm{Ti}_{5} \mathrm{O}_{12} / \mathrm{C}$ anode material with ultrafast charge/discharge capability for lithium ion batteries. J. Alloys Compd., 2016, 671: 157-163.

[52] KUO Y C, LIN J Y. One-pot Sol-Gel synthesis of $\mathrm{Li}_{4} \mathrm{Ti}_{5} \mathrm{O}_{12} / \mathrm{C}$ anode materials for high-performance Li-ion batteries. Electrochim. Acta, 2014, 142: 43-50.

[53] GAO L, LIU R J, HU H, et al. Carbon-decorated $\mathrm{Li}_{4} \mathrm{Ti}_{5} \mathrm{O}_{12} /$ rutile $\mathrm{TiO}_{2}$ mesoporous microspheres with nanostructures as high-performance anode materials in lithium-ion batteries. Nanotechnology, 2014, 25(17): 175402-175410.

[54] XUE R, YAN J W, JIANG L, et al. Fabrication of lithium titanate/ graphene composites with high rate capability as electrode materials for hybrid electrochemical super capacitors. Mater. Chem. Phys., 2015, 160: 375-382.

[55] LI R Y, CHEN T Y, SUN B B, et al. Novel lithium titanate-graphene hybrid containing two graphene conductive frameworks for lithiumion battery with excellent electrochemical performance. Mater. Res. Bull., 2015, 70: 965-975.

[56] YANG X J, ZHENG A B, WANG X L, et al. Graphene nanosheet and carbon layer co-decorated $\mathrm{Li}_{4} \mathrm{Ti}_{5} \mathrm{O}_{12}$ as high performance anode material for rechargeable lithium-ion batteries. Ceram. Int., 2017, 43(3): 3252-3258.

[57] KIM J, LEE K E, KIM K H, et al. Single-layer graphene-wrapped $\mathrm{Li}_{4} \mathrm{Ti}_{5} \mathrm{O}_{12}$ anode with superior lithium storage capability. Carbon, 2017, 114: 275-283.

[58] KIM K T, YU C Y, YOON C S, et al. Carbon-coated $\mathrm{Li}_{4} \mathrm{Ti}_{5} \mathrm{O}_{12}$ nanowires showing high rate capability as an anode material for rechargeable sodium batteries. Nano Energy, 2015, 12: 725-734.

[59] LUO H J, SHEN L F, RUI K, et al. Carbon coated $\mathrm{Li}_{4} \mathrm{Ti}_{5} \mathrm{O}_{12}$ nanorods as superior anode material for high rate lithium ion batteries. $J$. Alloys Compd., 2013, 572: 37-42.

[60] LIU J, SONG K, AKEN P A, et al. Self-supported $\mathrm{Li}_{4} \mathrm{Ti}_{5} \mathrm{O}_{12}-\mathrm{C}$ nanotube arrays as high-rate and long-life anode materials for flexible Li-ion batteries. Nano Lett., 2014, 14(5): 2597-2603. 